\title{
A quantitative and thermodynamically consistent phase-field interpolation function for multi-phase systems
}

\author{
Nele Moelans* \\ Dept. Metallurgy and Materials Engineering, Katholieke Universiteit Leuven, \\ Kasteelpark Arenberg 44, bus 24.50, B-3001 Leuven, Belgium
}

\begin{abstract}
The aimed properties of the interpolation functions used in quantitative phase-field models for 2-phase systems do not extend to multi-phase systems. Therefore, a new type of interpolation functions is introduced, which has a zero-slope at the equilibrium values of the non-conserved field variables representing the different phases and allows for a thermodynamically consistent interpolation of the free energies. The interpolation functions are applicable for multi-phase-field and multi-order-parameter representations and can be combined with existing quantitative approaches for alloys. A model for polycrystalline, multi-component and multi-phase systems is formulated using the new interpolation functions that accounts in a straightforward way for composition dependent expressions of the bulk Gibbs energies and diffusion mobilities and interfacial free energies and mobilities. The numerical accuracy of the approach is analyzed for coarsening and diffusion controlled parabolic growth in $\mathrm{Cu}-\mathrm{Sn}$ systems as a function of $R / \ell$, with $R$ grain size
\end{abstract}

\footnotetext{
*nele.moelans@mtm.kuleuven.be
} 
and $\ell$ diffuse interface width.

Keywords: phase-field method, multicomponent, multiphase, coarsening, growth kinetics

$P A C S$ :

\section{Introduction}

Many materials are multicomponent alloys and consist of several grains and phases. Their macroscopic properties and reliability highly depend on the microstructure and its evolution in time. It has been shown extensively that the phase-field technique is capable of dealing in a phenomenological, and more and more also quantitative, way with the complex processes and morphologies involved in microstructure evolution [1, 2, 3, 4, 5, 6].

To allow for quantitative 3D microstructure simulations on meso and micro length scales, thin-interface phase-field models have been developed which allow us to increase the diffuse interface width to decrease the computational cost without affecting other system properties. A first step in the development of quantitative phase-field models was the decoupling of bulk and interfacial free energy as proposed by Tiaden et al. [7, 8] and Kim et al. [9] for multi-component alloys and by Karma and Rappel [10] for pure materials. A next step was the addition of a non-variational anti-trapping current to decouple bulk and interface kinetics, introduced by Karma [1] and worked out by Echebarria et al. [12] and Ramirez et al. [13] for binary dilute alloys with negligible diffusion in the solid. The anti-trapping current has been generalized to non-dilute multi-component alloys by Kim [14].

The interpolation functions introduced for 2-phase systems, however, do 
not generalize to multi-phase systems. The function $h(\phi)=\phi^{3}(10-15 \phi+$ $\left.6 \phi^{2}\right)$, for example, is frequently used to interpolate between the bulk free energies of a liquid and solid phase $f_{L}$ and $f_{S}$ (expressed in $\mathrm{J} / \mathrm{m}^{2}$ ), giving $f_{b}=h(\phi) f_{L}+(1-h(\phi)) f_{S}$ (with $\phi=1$ representing the liquid and $\phi=0$ the solid). A straightforward generalization to multi-phase-field models, e.g. that of Steinbach et al. [15] with $\phi_{i}=1$ and $\phi_{j}=0, \forall j \neq i$ representing phase $i$ and $\sum_{i} \phi_{i}=1$, would give a bulk free energy of the form $f_{b}=$ $\sum h_{i}\left(\phi_{i}\right) f^{i}$ which is thermodynamically inconsistent since $\sum_{i} h_{i}\left(\phi_{i}\right) \neq 1$ in multi-junctions. Often an interpolation function of the form $h_{i}\left(\phi_{i}\right)=\phi_{i}$ is therefore used in multi-phase-field models [6], although it shifts the local minima of the total free energy and may thus introduce inaccuracies, which are difficult to control.

Folch and Plapp [16, 17] have formulated a free energy functional for 3phase systems with interpolation functions $g_{i}\left(\phi_{1}, \phi_{2}, \phi_{3}\right), i=1 \ldots 3$ that are polynomials of fifth order in the phase-fields. The interpolation functions allow for a thermodynamic consistent interpolation between the free energies of the 3 coexisting phases and keep the local minima of the free energy at their intended positions. They have also shown, however, that in order to satisfy all requirements for a quantitative phase-field model, the order of the interpolation polynomial has to increase with the number of phases, making a generalization to multi-phase structures practically unfeasible.

Besides multi-phase-field representations, multi-order-parameter representations are frequently used, for example that of Chen and Yang [18] for polycrystalline structures and that of Khachaturyan [19] for ordered precipitates. Order-parameters are not interpreted as fractions; consequently, their 
values do not necessarily sum up to 1 . Multi-order-parameter models have been combined with the thin-interface approach for multi-component alloys for example by Zhu et al. [20], and with the thin-interface approach for dilute binary alloys by Ofori-Opoku and Provatas 21. However, these approaches as well do not extend to multi-phase systems.

The purpose of this paper is to introduce and validate a new type of interpolation functions for quantitative phase-field modeling for multi-phase systems. In section 2, a quantitative phase-field model for coarsening and diffusion controlled growth in polycrystalline multi-component and multi-phase systems is formulated using the new interpolation functions and relations between the model parameters and physical properties are derived. The quantitative accuracy of the approach is validated for 4 basic microstructural configurations involving coarsening and diffusion controlled parabolic growth in section 3. In section 4, the main findings of the presented work are summarized and further applications for the interpolation functions are mentioned. The numerical validation is limited to binary systems with a parabolic composition dependence of the bulk free energies, to allow for simple criterions that can be used as a rule of thumb when designing a phase-field simulation. Further validation for multi-component systems with arbitrary composition dependence is in progress. The parameter space becomes however much larger and conclusions are more complex. 


\section{Model and multi-phase interpolation functions}

\subsection{Variables, conditions and free energy functional}

A system of $C$ components $(k=1 \ldots C)$ and $n$ phases $(\rho=\alpha, \beta, \ldots)$ is considered. The pressure, temperature and molar volume of the system are assumed to be constant. For each phase $\rho, p_{\rho}$ crystallographic orientations are distinguished $\left(i=1 \ldots p_{\rho}\right)$, with $p_{\rho}$ infinite in the limit of a continuous grain orientation representation, but practically determined by the resolution with which grain orientations are represented [22]. Driving forces and gradients in properties are considered to be relatively small, such that the assumption of local thermodynamic equilibrium is applicable.

The phases and grains are represented by a set of non-conserved orderparameter fields, which are function of time $t$ and spatial coordinates $\mathbf{r}$,

$$
\begin{aligned}
\boldsymbol{\eta}= & \left(\eta_{\alpha 1}(\mathbf{r}, t), \eta_{\alpha 2}(\mathbf{r}, t), \ldots, \eta_{\alpha p_{\alpha}}(\mathbf{r}, t), \eta_{\beta 1}(\mathbf{r}, t), \ldots, \eta_{\beta p_{\beta}}(\mathbf{r}, t),\right. \\
& \left.\ldots, \eta_{\rho 1}(\mathbf{r}, t), \ldots, \eta_{\rho p_{\rho}}(\mathbf{r}, t) \ldots\right) \\
= & \left(\boldsymbol{\eta}_{\alpha}, \boldsymbol{\eta}_{\beta}, \ldots, \boldsymbol{\eta}_{\rho}, \ldots\right) .
\end{aligned}
$$

The first symbol in the indices, $\alpha, \beta, \ldots, \rho, \ldots$, refers to the different phases and the second, $i=1 \ldots p_{\rho}$, to the different crystallographic orientations of each phase. It is assumed that a particular "phase structure/orientation" combination " $\rho, i$ " is represented by the combination of order-parameter values in which $\eta_{\rho, i}=1$ and $\eta_{\sigma, j}=0$ for $[\sigma, j] \neq[\rho, i]$. Across the grain interfaces, the order-parameters change value smoothly and monotonously.

Moreover, conserved fields representing the local molar fractions of $C-1$ independent components are used

$$
\boldsymbol{x}=\left(x_{1}(\mathbf{r}, t), x_{2}(\mathbf{r}, t), \ldots, x_{k}(\mathbf{r}, t), \ldots x_{C-1}(\mathbf{r}, t)\right) .
$$


The total free energy $F_{\text {tot }}$ of the system is formulated as a functional of the order-parameter fields $\boldsymbol{\eta}$ and molar-fraction fields $\boldsymbol{x}$, as

$$
F_{\text {tot }}(\boldsymbol{\eta}, \boldsymbol{x})=F_{s}(\boldsymbol{\eta}, \boldsymbol{x})+F_{b}(\boldsymbol{\eta}, \boldsymbol{x})=\int_{V} f_{s}(\boldsymbol{\eta}, \boldsymbol{x})+f_{b}(\boldsymbol{\eta}, \boldsymbol{x}) \mathrm{d} V(3)
$$

with decoupled contributions for bulk $\left(f_{b}\right)$ and interfacial $\left(f_{s}\right)$ free energy. The interfacial free energy for polycrystalline structures recently introduced by the author [23, 24] is extended to multi-phase systems in section 2.2. In section 2.3, a bulk free energy is derived, starting from the thin-interface approach of Tiaden et al. [7, 8] and Kim et al. [9], but using a different type of functions to interpolate between the free energies of the different phases.

\subsection{Interfacial free energy functional}

Applying the free energy functional derived in [23, 24] for the full set of order-parameter fields (1) gives

$$
f_{s}=m f_{0}(\boldsymbol{\eta})+\frac{\kappa(\boldsymbol{\eta})}{2} \sum_{\rho} \sum_{i=1}^{p_{\rho}}\left(\nabla \eta_{\rho i}\right)^{2},
$$

with $f_{0}$ a fourth-order Landau polynomial of the order-parameters

$f_{0}(\boldsymbol{\eta})=\sum_{\rho} \sum_{i=1}^{p_{\rho}}\left[\frac{\eta_{\rho i}^{4}}{4}-\frac{\eta_{\rho i}^{2}}{2}\right]+\sum_{\rho} \sum_{i=1}^{p_{\rho}}\left[\sum_{\sigma} \sum_{j=1, \rho i \neq \sigma j}^{p_{\sigma}} \frac{\gamma_{\rho i, \sigma j}\left(\psi_{\rho i, \sigma j}, \widetilde{\boldsymbol{\mu}}\right)}{2} \eta_{\rho i}^{2} \eta_{\sigma j}^{2}\right]+\frac{1}{4}$

and where $\sum_{\rho} \sum_{i}$ indicates a sum over all possible orientations of all phases and $\sum_{\rho} \sum_{i}\left[\sum_{\sigma} \sum_{j, \rho i \neq \sigma j}\right]$ the sum over all possible combinations of orderparameters twice, excluding those with two times the same order-parameter. The function $f_{0}$ is minimal and equals zero for $\left(\ldots, \eta_{\rho, i}, \ldots\right)=\ldots,(\ldots, 0,1,0, \ldots), \ldots$, corresponding to all possible "phase structure/orientation" combination " $\rho$ ". 
The gradient coefficient $\kappa$ is formulated as a function of the order-parameters as proposed by Kazaryan et al. [25]

$$
\kappa(\boldsymbol{\eta})=\frac{\sum_{\rho} \sum_{i=1}^{p_{\rho}}\left[\sum_{\sigma} \sum_{j=1, \sigma j \neq \rho i}^{p_{\sigma}} \kappa_{\rho i, \sigma j}\left(\psi_{\rho i, \sigma j}, \widetilde{\boldsymbol{\mu}}\right) \eta_{\rho i}^{2} \eta_{\sigma j}^{2}\right]}{\sum_{\rho} \sum_{i=1}^{p_{\rho}}\left[\sum_{\sigma} \sum_{j=1, \sigma j \neq \rho i}^{p_{\sigma}} \eta_{\rho i}^{2} \eta_{\sigma j}^{2}\right]}
$$

Assuming that at each interface only the 2 order-parameters representing the adjacent grains differ from 0 (This is true if the dependence of the model parameter $\kappa$ on $\eta_{\rho i}$ is ignored when deriving $\delta \mathrm{F}_{s} / \delta \eta_{\rho i}$ in equations (16) (see also [5], section III E) or for $\left.\kappa_{i, j}=\kappa, \forall i, j.\right)$, it can be verified that at the interface between grains ' $\rho i$ ' and ' $\sigma j$ ', $\kappa=\kappa_{\rho i, \sigma j}$ in equation (6) and only the term $\gamma_{\rho i, \sigma j} \eta_{\rho i}^{2} \eta_{\sigma j}^{2}$ remains in the sum of cross products in equation (5). The free energy and width of an interface ' $\rho i, \sigma j$ ' is thus determined by $\gamma_{\rho i, \sigma j}$ and $\kappa_{\rho i, \sigma j}$. Inclination dependence of the interfacial free energy can be introduced by formulating the model parameters $\gamma_{\rho i, \sigma j}\left(\psi_{\rho i, \sigma j}\right)$ and $\kappa_{i, j}\left(\psi_{\rho i, \sigma j}\right)$ as a function of the direction of the normal to the interface [25, 24], but is not considered further in this paper. Since the thin interface formulation introduced in the next section dictates that, at each position, the diffusion potentials are equal in all phases (Equation (8)), it is most convenient to introduce composition dependence of the interfacial properties by formulating the model parameters as a function of the local diffusion potentials, namely $\gamma_{\rho i, \sigma j}(\widetilde{\boldsymbol{\mu}})$ and $\kappa_{\rho i, \sigma j}(\widetilde{\boldsymbol{\mu}})$.

\subsection{Chemical bulk free energy functional and new interpolation functions}

Following the thin-interface approach for multi-component alloys as described in [7, 9, 8, 26, 14, 6], interfaces are treated as a mixture of 2 phases, each with its own phase-composition $\boldsymbol{x}_{\rho}$ and composition dependent free 
energy function $f_{\rho}\left(\boldsymbol{x}_{\rho}\right)$. The bulk free energy $f_{b}$ has a form

$$
f_{b}=\sum_{\rho} h_{\rho} f_{\rho}\left(\boldsymbol{x}_{\rho}\right)=\sum_{\rho} h_{\rho} \frac{G_{\rho, m}\left(\boldsymbol{x}_{\rho}\right)}{V_{m}},
$$

with $\sum_{\rho}$ the sum over all phases, $f_{\rho}$ the energy density and $G_{\rho, m}$ the molar Gibbs energy of phase $\rho$. The interpolation functions $h_{\rho}$ must have a zero slope at the equilibrium values of the non-conserved phase-fields or orderparameters representing the different phases and $\sum_{\rho} h_{\rho}$ must equal 1 , for example $h(\phi)=\phi^{3}\left(10-15 \phi+6 \phi^{2}\right)$ for a 2 -phase system with equilibrium values $\phi=1$ and $\phi=0$ in the bulk phases. The phase-compositions $\boldsymbol{x}_{\rho}$ are determined so that at each position the diffusion potentials of all components in all coexisting phases $\widetilde{\mu}_{\rho, k}=\partial f_{\rho}\left(\boldsymbol{x}_{\rho}\right) / \partial x_{\rho, k}$ are equal, giving the following set of equations

$$
\frac{\partial f_{\alpha}\left(\boldsymbol{x}_{\alpha}\right)}{\partial x_{\alpha, k}}=\frac{\partial f_{\beta}\left(\boldsymbol{x}_{\beta}\right)}{\partial x_{\beta, k}}=\ldots=\frac{\partial f_{\rho}\left(\boldsymbol{x}_{\rho}\right)}{\partial x_{\rho, k}}=\widetilde{\mu}_{k}, \quad \forall k,
$$

and so that the overall composition $\boldsymbol{x}$ relates to the phase-compositions as

$$
\boldsymbol{x}=\sum_{\rho} h_{\rho} \boldsymbol{x}_{\rho}
$$

Within the bulk of phase $\rho, \boldsymbol{x}_{\rho}=\boldsymbol{x}$ and $f_{b}=f_{\rho}(\boldsymbol{x})$. Only, at interfaces, the composition profile and local free energy are affected by the thininterface formulation. An important advantage of the thin-interface approach (7)-(9) compared to other quantitative alloy approaches is that composition dependent Gibbs energy expressions for multi-component systems optimized according to the CALPHAD approach [27] can be used directly in the phasefield bulk energy (7) [28, 29, 30, 31, 32, 33]. The formalism has been extended to systems with interstitial elements by Cha et al. [34. 
For multi-phase systems, we introduce the following interpolation functions for a multi-order-parameter representation (1):

$$
h_{\alpha}(\boldsymbol{\eta})=\phi_{\alpha}(\boldsymbol{\eta})=\frac{\sum_{i=1}^{p_{\alpha}} \eta_{\alpha i}^{2}}{\sum_{\rho} \sum_{i=1}^{p_{\rho}} \eta_{\rho i}^{2}}=\frac{\left|\boldsymbol{\eta}_{\alpha}\right|^{2}}{\sum_{\rho}\left|\boldsymbol{\eta}_{\rho}\right|^{2}}
$$

for phase $\alpha$ and analogous expressions for the other phases. Since $\sum_{\rho} \phi_{\rho}=1$, the $\phi_{\alpha}$ can be interpreted as phase-fractions. Furthermore, it can be verified that $\forall \alpha, \rho, \mathrm{d} \phi_{\alpha} / \mathrm{d} \eta_{\rho i}\left(\eta_{\rho i}=0\right)=\mathrm{d} \phi_{\alpha} / \mathrm{d} \eta_{\rho i}\left(\eta_{\rho i}=1, \eta_{\sigma j, \sigma j \neq \rho i}=0\right)=0$ (see figure 1]. Similarly, for a multi-phase-field representation $\breve{\phi}_{\alpha}, \breve{\phi}_{\beta}, \ldots$ with $\sum_{\rho} \breve{\phi}_{\rho}=1$, interpolation functions of the form

$$
h_{\alpha}\left(\breve{\phi}_{\alpha}, \breve{\phi}_{\beta}, \ldots\right)=\phi_{\alpha}=\frac{\breve{\phi}_{\alpha}^{2}}{\sum_{\rho} \breve{\phi}_{\rho}^{2}}
$$

can be formulated and it can be verified, now considering $\sum_{\rho} \breve{\phi}_{\rho}=1$, that again $\forall \alpha, \rho, \mathrm{d} \phi_{\alpha} / \mathrm{d} \breve{\phi}_{\rho}\left(\breve{\phi}_{\rho}=0\right)=\mathrm{d} \phi_{\alpha} / \mathrm{d} \breve{\phi}_{\rho i}\left(\breve{\phi}_{\rho}=1, \breve{\phi}_{\sigma, \sigma \neq \rho}=0\right)=0$.

The interpolation functions $(10)$ and (11) meet the requirements to be combined with other thin-interface approaches for alloys as well. They can be used in the bulk free energy formulation of Folch and Plapp [17] instead of the fifth order polynomials, giving

$$
f_{b}=\frac{1}{2}\left(x_{B}-\sum_{\rho} A_{\rho}(T) h_{\rho}\left(\breve{\phi}_{\alpha}, \ldots, \breve{\phi}_{\rho}, \ldots\right)\right)^{2}+\sum_{\rho} B_{\rho}(T) h_{\rho}\left(\breve{\phi}_{\alpha}, \ldots, \breve{\phi}_{\rho}, \ldots\right)
$$

A generalization towards multi-phase systems is now straightforward. In principle, it is also possible to take the interpolation functions $\widetilde{g}$ and $\bar{g}$ in the bulk free energy for binary dilute alloys of Echebarria et al. [12] as $\widetilde{g}=\phi_{\alpha}$ and $\bar{g}=\frac{1}{\ln (k)} \ln \left[1-(1-k) \phi_{\alpha}\right][21]$ with $k=x_{\alpha, \text { eq }} / x_{\beta, \text { eq }}$ the ratio between the equilibrium concentrations in the coexisting phases $\alpha$ and $\beta$. For multi-phase 
systems, it is however complicated to find an appropriate relation between the 2 interpolation functions $\bar{g}\left(\widetilde{g}_{\rho}\right)$.

The new interpolation functions can be used to extend the classical WBM phase-field approach for alloy solidification [35] to multi-phase systems, resulting in a bulk free energy of the form

$$
f_{b}=\sum_{\rho} h_{\rho} f_{\rho}(\boldsymbol{x})=\sum_{\rho} h_{\rho} \frac{G_{\rho, m}(\boldsymbol{x})}{V_{m}},
$$

where the free energies of the different phases are evaluated for the actual local composition. A bulk free energy density of the form (13) however contributes to the interfacial energy making a general quantitative approach on realistic length scales computationally unfeasible.

\subsection{Mass diffusion and interface movement}

The diffusion equations of the $C-1$ independent components are of the form

$$
\frac{\partial x_{k}}{\partial t}=-\nabla \cdot J_{k}=\nabla \cdot \sum_{m=1}^{C-1}\left[M_{k m}(\boldsymbol{\eta}, \boldsymbol{x}) \nabla \frac{\delta F(\boldsymbol{\eta}, \boldsymbol{x})}{\delta x_{m}}\right],
$$

with $J_{k}$ the flux of component $k$ and usually $M_{k m}(\boldsymbol{\eta}, \boldsymbol{x})=\sum_{\rho} \phi_{\rho} M_{\rho, k m}(\boldsymbol{x})$ [9, 14, giving

$$
\begin{aligned}
J_{k} & =-\sum_{m=1}^{C-1}\left[M_{k m}(\boldsymbol{\eta}, \boldsymbol{x}) \nabla \sum_{\rho} \phi_{\rho} \frac{\partial f_{\rho}\left(\boldsymbol{x}_{\rho}\right)}{\partial x_{\rho, m}} \frac{\partial x_{\rho, m}}{\partial x_{m}}\right] \\
& =-\left[\sum_{m=1}^{C-1}\left[\sum_{\rho} \phi_{\rho} M_{\rho, k m}(\boldsymbol{x})\right] \nabla \widetilde{\mu}_{m}\right] .
\end{aligned}
$$

Within the bulk of each phase, equation (15) reduces to an Onsager type of diffusion equation with $M_{\rho, k m}$ the mobilities of phase $\rho$ for interdiffusion in a 
volume fixed frame [36]. The $M_{\rho, k m}$ are related to the atomic mobilities of the different elements $\Omega_{\rho, k}(k=1 \ldots C)$ as $M_{\rho, k m}=\sum_{l=1}^{C}\left(\delta_{k l}-x_{k}\right)\left(\delta_{m l}-x_{m}\right) x_{l} \Omega_{\rho, l}$ and to the chemical (inter)diffusivities as $D_{\rho, k m}=\sum_{l=1}^{C-1} M_{\rho, k l}\left(\partial^{2} f / \partial x_{l} \partial x_{m}\right)$ [36, 37, 38, 32, 39]. Composition dependent expressions for the atomic mobilities optimized according to the CALPHAD approach [40] and diffusion coefficients calculated from first principles [41, 42, 43, 44] can thus be used directly in the diffusion equations (14)- 15 .

The evolution of the non-conserved order-parameter fields $\eta_{\rho i}$ follows

$$
\begin{aligned}
\frac{\partial \eta_{\rho i}}{\partial t}=- & -L(\boldsymbol{\eta}) \frac{\delta F(\boldsymbol{\eta}, \boldsymbol{x})}{\delta \eta_{\rho i}}=-L(\boldsymbol{\eta})\left(\eta_{\rho i}^{3}-\eta_{\rho i}\right. \\
& +2 \eta_{\rho i} \sum_{\sigma} \sum_{j=1, \sigma j \neq \rho i}^{p_{\sigma}} \gamma_{\rho i, \sigma j} \eta_{\sigma j}^{2}-\kappa(\boldsymbol{\eta}) \nabla^{2} \eta_{\rho i} \\
+ & \frac{2 \eta_{\rho i}}{\sum_{\sigma}\left|\boldsymbol{\eta}_{\sigma}\right|^{2}}\left[\left[1-\phi_{\rho}\right]\left[f_{\rho}\left(\boldsymbol{x}_{\rho}\right)-\sum_{k=1}^{C-1} \widetilde{\mu}_{k} x_{\rho, k}\right]\right. \\
& \left.\left.-\sum_{\sigma \neq \rho} \phi_{\sigma}\left[f_{\sigma}\left(\boldsymbol{x}_{\sigma}\right)-\sum_{k=1}^{C-1} \widetilde{\mu}_{k} x_{\sigma, k}\right]\right]\right) .
\end{aligned}
$$

with the kinetic coefficient $L(\boldsymbol{\eta})$

$$
L(\boldsymbol{\eta})=\frac{\sum_{\rho i} \sum_{\sigma j \neq \rho i}^{p} L_{\rho i, \sigma j}\left(\widetilde{\boldsymbol{\mu}}, \psi_{\rho i, \sigma j}\right) \eta_{\rho i}^{2} \eta_{\sigma j}^{2}}{\sum_{\rho i} \sum_{\sigma j \neq \rho i} \eta_{\rho i}^{2} \eta_{\sigma j}^{2}}
$$

To avoid third-phase contributions [24] and interface width dependent solute segregation at interfaces, interface properties are treated as fixed for a given grain configuration and local diffusion potential (similar as variations in grain boundary energy with misorientation are treated in Monte Carlo Potts approaches [45]). The $\eta$ - and $x$-dependence of $\kappa$ and $\gamma$ are therefore 
not considered when taking the derivative in equations (14) and (16). The limitations of such a not-fully-variational approach are discussed in section 3. The equations can be fully variational, when taking all $\gamma_{\rho i, \sigma j}$ independent of composition and all $\kappa_{\rho i, \sigma j}=\kappa$ equal and independent of composition.

\subsection{Parameter relations for interfacial properties}

The specific free energy $\left(\mathrm{J} / \mathrm{m}^{2}\right)$ of a diffuse interface between two phases $\alpha$ and $\beta$ in chemical equilibrium is defined as [46],

$$
\begin{aligned}
\sigma_{\alpha, \beta}= & F_{\mathrm{tot}}-\int_{-\infty}^{0} f_{\alpha}\left(\boldsymbol{x}_{\alpha, \mathrm{eq}}\right) \mathrm{dx}-\int_{0}^{+\infty} f_{\beta}\left(\boldsymbol{x}_{\beta, \mathrm{eq}}\right) \mathrm{dx} \\
= & \int_{-\infty}^{+\infty} f_{s}\left(\eta_{\alpha}, \eta_{\beta}, \widetilde{\boldsymbol{\mu}}_{\mathrm{eq}}\right) \mathrm{dx} \\
& \quad+\left(f_{\alpha}\left(\boldsymbol{x}_{\alpha, \mathrm{eq}}\right)-f_{\beta}\left(\boldsymbol{x}_{\beta, \mathrm{eq}}\right)\right)\left[\int_{-\infty}^{0} \phi_{\beta} \mathrm{dx}-\int_{0}^{+\infty}\left[1-\phi_{\beta}\right] \mathrm{dx}\right] \\
= & \int_{-\infty}^{+\infty} f_{s}\left(\eta_{\alpha}, \eta_{\beta}, \widetilde{\boldsymbol{\mu}}_{\mathrm{eq}}\right) \mathrm{dx}+0
\end{aligned}
$$

with $\mathrm{x}$ measured along the normal to the interface and $\boldsymbol{x}_{\alpha, \text { eq }}, \boldsymbol{x}_{\beta, \text { eq }}$ and $\widetilde{\boldsymbol{\mu}}_{\text {eq }}$ the equilibrium compositions of the coexisting phases and equilibrium diffusion potential of the system. Moreover, it is used that $\phi_{\alpha}+\phi_{\beta}=1$ for an interface between phases $\alpha$ and $\beta$. The bulk free energy contribution (7) has accordingly no effect on the interfacial free energy and the shape of the stationary $\eta$-profiles across the interface. The parameter relations for the interfacial free energy $\sigma_{\alpha, \beta}$ and interface width $\ell_{\alpha, \beta}$ derived for a polycrystalline single-phase system in [24] thus remain valid, giving

$$
\begin{aligned}
\sigma_{\alpha, \beta}(\widetilde{\boldsymbol{\mu}}) & =g\left(\gamma_{\alpha, \beta}(\widetilde{\boldsymbol{\mu}})\right) \sqrt{m \kappa_{\alpha, \beta}(\widetilde{\boldsymbol{\mu}})} \\
\ell_{\alpha, \beta}(\widetilde{\boldsymbol{\mu}}) & =\sqrt{\frac{\kappa_{\alpha, \beta}(\widetilde{\boldsymbol{\mu}})}{m f_{0, \max }\left(\gamma_{\alpha, \beta}(\widetilde{\boldsymbol{\mu}})\right)}},
\end{aligned}
$$


where the function $g\left(\gamma_{\alpha, \beta}\right)$ refers to an integral along the order-parameter profiles and $f_{0, \max }\left(\gamma_{\alpha, \beta}\right)$ is evaluated from 5 for $\eta_{\alpha}^{0}(\mathrm{x}=0)=\eta_{\beta}^{0}(\mathrm{x}=0)$, with $\eta_{\alpha}^{0}$ and $\eta_{\beta}^{0}$ the stationary profiles. The functions have been evaluated numerically over a large range of $\gamma$-values [47]. The interface width $\ell$ is defined based on the steepest gradient so that an equal interface width results in equal accuracy and stability criteria in the numerical solution of the phase-field equations. Furthermore, for curvature driven movement of a grain boundary between 2 grains of the same phase, the kinetic coefficient $L_{\alpha 1, \alpha 2}$ in equation (16) relates to grain boundary mobility $m_{\alpha 1, \alpha 2}$ as

$$
m_{\alpha 1, \alpha 2}(\widetilde{\boldsymbol{\mu}})_{\alpha 1, \alpha 2}(\widetilde{\boldsymbol{\mu}})=\kappa_{\alpha 1, \alpha 2}(\widetilde{\boldsymbol{\mu}}) L_{\alpha 1, \alpha 2}(\widetilde{\boldsymbol{\mu}}),
$$

with $\sigma_{\alpha 1, \alpha 2}(\widetilde{\boldsymbol{\mu}})$ and $m_{\alpha 1, \alpha 2}(\widetilde{\boldsymbol{\mu}})$ an effective grain boundary energy and mobility which account implicitly for the effects of solute segregation at the grain boundary as in [48].

\subsection{Thin-interface approach for diffusion controlled interface movement}

The new interpolation functions (10) meet all requirements that follow from a thin-interface analysis for alloys [12, 14]. For $\ell v_{n} / \widetilde{D} \ll 1$ and $\ell / R_{1 / 2} \ll$ 1 , with $v_{n}$ interface velocity, $R_{1}$ and $R_{2}$ the principle radii of curvature and $\widetilde{D}$ the average diffusivity at the interface, it is therefore obtained that [14]

$$
\begin{aligned}
& f_{\alpha}\left(\boldsymbol{x}_{\alpha, \mathrm{eq}}\right)-f_{\beta}\left(\boldsymbol{x}_{\beta, \mathrm{eq}}\right)-\sum_{k=1}^{C-1}\left(x_{\alpha, \mathrm{eq}, k}-x_{\beta, \mathrm{eq}, k}\right) \widetilde{\mu}_{k, i}=\Delta f_{i}^{\alpha \rightarrow \beta} \\
= & \sigma_{\alpha, \beta}\left(\frac{1}{R_{1}}+\frac{1}{R_{2}}\right)+v_{n}\left[\sqrt{\frac{m}{\kappa_{\alpha, \beta}}} \frac{g\left(\gamma_{\alpha, \beta}\right)}{L_{\alpha, \beta}}-\sqrt{\frac{\kappa_{\alpha, \beta}}{2 m}} I_{\phi}\left(\gamma_{\alpha, \beta}\right) \zeta\right],
\end{aligned}
$$

where $\widetilde{\mu}_{k, i}$ is the diffusion potential at which the new phase forms and $\zeta=$ $\Delta \mathbf{c M}^{-1} \boldsymbol{\Delta} \mathbf{c}=\sum_{k=1}^{C-1}\left(x_{\alpha, \mathrm{eq}, k}-x_{\beta, \mathrm{eq}, k}\right) \sum_{m=1}^{C-1} m_{k m}\left(x_{\alpha, \mathrm{eq}, m}-x_{\beta, \mathrm{eq}, m}\right)$, with $m_{k m}$ 
the elements of the inverse of the diffusion mobilities matrix $\mathbf{M}=M_{m k}$. For the interpolation functions $(10)$, the integral $I_{\phi}$ equals

$$
I_{\phi}\left(\gamma_{\alpha, \beta}\right)=\sqrt{\frac{2 m}{\kappa_{\alpha, \beta}}} \int_{-\infty}^{+\infty} \phi_{\alpha}^{0}\left(1-\phi_{\alpha}^{0}\right) \mathrm{dx},
$$

with $\phi_{\alpha}^{0}$ evaluated for the stationary order-parameter profiles. Numerically evaluated function values of $I_{\phi}(\gamma)$ are listed at [47].

Matching the thin-interface approximation (23) with the sharp interface equation for diffusive transformations $\Delta f_{i}^{\alpha \rightarrow \beta}=\sigma_{\alpha, \beta}\left(1 / R_{1}+1 / R_{2}\right)+v_{n} / m_{\alpha, \beta}$ [49, 50], relates the kinetic coefficient $L_{\alpha, \beta}$ in the phase-field model to the interface mobility $m_{\alpha, \beta}$. Therefore, bulk diffusion controlled growth with infinite reaction rate at the interface $\left(1 / m_{\alpha, \beta}=0\right)$ is obtained in the phasefield model using

$$
L_{\alpha, \beta}=L_{\alpha, \beta}^{\operatorname{leq}(\widetilde{\boldsymbol{\mu}})}=\frac{\sqrt{2} m g\left(\gamma_{\alpha, \beta}(\widetilde{\boldsymbol{\mu}})\right)}{\kappa_{\alpha, \beta}(\widetilde{\boldsymbol{\mu}}) I_{\phi}\left(\gamma_{\alpha, \beta}(\widetilde{\boldsymbol{\mu}})\right) \zeta}
$$

For $L<L_{\alpha, \beta}^{\text {leq }}$, the $\eta$-profiles will not adapt instantaneously for changes in the molar-fraction profiles due to bulk diffusion, whereas for $L>L_{\alpha, \beta}^{\text {leq }}$, the $\eta$ profiles will become unstable and break free from the molar-fraction profiles.

The thin-interface analysis resulting in equation (23) requires $M_{\alpha, m k}=$ $M_{\beta, m k}=M_{m k}(2$-sided model $)$ or, for $\alpha$ the growing phase and with a nonvariational antitrapping current in the diffusion equations, $M_{\alpha, m k}=0$ and $M_{k m}=M_{\beta, m k}$ (one-sided model). The anti-trapping concept [11, 12] has been extended to multi-phase systems and systems with arbitrary thermodynamic and diffusion properties [51, 6] and to multi-order-parameter representations [21] for polycrystalline solidification. The new interpolation functions have the appropriate form to be combined with these approaches. There is, however, no full solution yet that removes all exaggerated non-equilibrium effects 
[52] in the phase-field model for the general case $M_{\alpha, m k} \neq M_{\beta, m k} \neq 0$ important for multi-phase systems. The simulations discussed in section 3 show that for coarsening and parabolic growth, the non-equilibrium effects are negligible without anti-trapping current even when using a mesoscale interface width. Under these conditions, result (25) with $M_{m k}=0.5\left(M_{\alpha, m k}+M_{\beta, m k}\right)$ can still be used to calculate the value of $L_{\alpha, \beta}$ for which growth is close to bulk diffusion controlled growth.

\section{Numerical validation}

The effect of the diffuse interface width and grid spacing on the accuracy with which the analytical parameter relations are reproduced in the simulations, was validated for 4 microstructural configurations, namely a stagnated triple junction, the growth of an intermediate phase, the growth of a sherical grain from a supersaturated matrix and Ostwald ripening of 2 spherical grains. A binary $\mathrm{Cu}-\mathrm{Sn}$ system was therefor considered with 3 phases, namely a Cu-rich fcc phase, a Sn-rich bct phase and an intermediate phase $\mathrm{Cu}_{6} \mathrm{Sn}_{5}$, and a parabolic composition dependence of the form $f_{\rho}=A_{\rho} / 2\left(x_{\rho}-x_{\rho, 0}\right)^{2}+C_{\rho}$ was assumed for the bulk free energies of the phases, with $A_{\mathrm{fcc}}=1 \mathrm{e} 8, A_{\mathrm{bct}}=1 \mathrm{e} 9$ and $A_{\mathrm{Cu} 6 \mathrm{Sn} 5}=1 \mathrm{e} 9, C_{\mathrm{fcc}}=0, C_{\mathrm{bct}}=1 \mathrm{e} 7$ and $C_{\mathrm{Cu} 6 \mathrm{Sn} 5}=-1 \mathrm{e} 6$ and $x_{\mathrm{fcc}, 0}=0.076, x_{\mathrm{bct}, 0}=0.978$ and $x_{\mathrm{Cu} 6 \mathrm{Sn} 5,0}=0.455$. For these free energies, the common tangent rule gives $x_{\mathrm{fcc}, \mathrm{eq}}=0.050$ and $x_{\mathrm{Cu} 6 \mathrm{Sn} 5 \text {,eq }}=0.452$ for fcc coexisting with $\mathrm{Cu}_{6} \mathrm{Sn}_{5}$ and $x_{\mathrm{bct} \text {,eq }}=0.999$ and $x_{\mathrm{Cu} 6 \mathrm{Sn} 5, \mathrm{eq}}=0.476$ for bct coexisting with $\mathrm{Cu}_{6} \mathrm{Sn}_{5}$ which are close to the equilibrium compositions at $T=150^{\circ} \mathrm{C}$, ignoring the $\mathrm{Cu}_{3} \mathrm{Sn}$ phase. Furthermore, the diffusion mobilities relate to the diffusion coefficients as $M_{\rho}=D_{\rho} / A_{\rho}$. 
Unless indicated differently, the interfacial free energy $\sigma$ was taken $0.5 \mathrm{~J} / \mathrm{m}^{2}$ and the model parameter $\gamma=1.5$ for all interfaces, so that $g(\gamma)=\sqrt{2} / 3$, $f_{0}(\gamma)=1 / 8$ and $I_{\phi}(\gamma)=1 / 2$. The other model parameters were accordingly calculated as a function of interface width from $\kappa=3 / 4 \sigma \ell=3 / 8 \ell$, $m=6 \sigma / \ell=3 / \ell$ and $L^{\text {leq }}=(4 m) /(3 \kappa \zeta)$. A standard explicit finite difference scheme with 5-point stencil for the laplacian was used to discretize the equations. Neumann boundary conditions were applied. All simulations start from a molar-fraction field for Sn and order-parameter fields with sharp steps at the interfaces which become diffuse during the first time steps of the simulation. The main findings are presented in this section.

In table 1, angles obtained from simulations of a stagnated triple junction are listed for 3 different ratios of $\sigma_{12}$ to $\sigma_{13}$ and for different interface widths $\ell$ and grid spacings $\Delta \mathrm{x}$ (although the conclusions are based on simulations for a larger range of ratios $\sigma_{12}$ to $\sigma_{13}$ ). Since the half lamellar spacing is 5 $\mu \mathrm{m}$, the Gibbs-Thomson effect is negligible. For given interfacial energies, interface width and grid spacing, the angles are close to those obtained in simulations for single-phase systems [24]. The bulk free energy and composition field have thus indeed negligible effect on the accuracy with which triple junction angles are reproduced in simulations. A general difficulty with triple-junctions is that for the non-variational variant of equation (16) with constant interface width, angles outside the range $110^{\circ}-150^{\circ}$ are difficult to obtain, whereas using the variational variant with constant $\kappa$, all stable angles and also wetting, are reproduced accurately using the spatial resolution required to resolve the movement of the thinnest interface. The latter approach is however inefficient from a numerical point of view because of the 
variations in interface width with variations in interface free energy.

In figure 2 simulation results are presented for the growth of an intermediate $\mathrm{Cu}_{6} \mathrm{Sn}_{5}$ phase in between a $\mathrm{Cu}$-rich fcc and Sn-rich bct phase, with the bct phase supersaturated in $\mathrm{Cu}\left(x_{\text {init }}=0.95\right) . L=L^{\text {leq }}$ for both interfaces, calculated from equation (25) with $M_{m k}=0.5\left(M_{\mathrm{Cu} 6 \mathrm{Sn} 5, m k}+M_{m k \text {,fcc/bct }}\right)$. Growth is first controlled by diffusion in the bct phase followed by a regime where growth is dominated by diffusion through the intermediate $\mathrm{Cu}_{6} \mathrm{Sn}_{5}$ layer. It can be verified that for $x_{\text {init }}=0.95, \ell v_{n} / D \ll 1$ after a growth distance of less than $\ell$, since for $1 \mathrm{D}$ layer growth $v_{n} / D=0.5 \cdot 1.2^{2} \cdot\left[\left(x_{\mathrm{bct}, \text { init }}-\right.\right.$ $\left.\left.x_{\mathrm{bct}, \mathrm{eq}}\right)^{2}\right] /\left[\left(x_{\mathrm{Cu} 6 \mathrm{Sn} 5 \text {,eq }}-x_{\mathrm{bct}, \text { init }}\right)\left(x_{\mathrm{Cu} 6 \mathrm{Sn} 5 \text {,eq }}-x_{\mathrm{bct}, \mathrm{eq}}\right)\right] \cdot 1 / S \approx 0.005$, with $S$ the growth distance [49, 53]. The diffuse interface width $\ell$ is therefore limited by he initial intermediate layer thickness. For $T_{\text {init }} / 2>\ell$, both parabolic growth regimes, as well as the transition in between, are reproduced accurately. Except within the diffuse interface region, the composition profiles for different interface widths match. The deviation (introduced at start-up over a distance much smaller than $\ell$ ) on the intermediate layer thickness becomes less than $2 \%$ when $T / 2=2 \ell$ and less than $1 \%$ when $T / 2=4 \ell$. For this $1 \mathrm{D}$ geometry, the simulation results are independent of $\Delta \mathrm{x}$ for $\ell \geq 3.3 \Delta \mathrm{x}$.

Fitting of simulation data for steady-state growth controlled by diffusion through the intermediate layer as shown in figure 3 , gives for $L=L^{\text {leq }}$, $T=k \sqrt{t}$ with the parabolic growth constant $k=0.0045 \mu \mathrm{m} / \mathrm{s}^{1 / 2}$ independent of the interface width, even for $\ell>T_{\text {init }} / 2$. For $\ell>T_{\text {init }} / 2$, the transition towards parabolic growth is however unrealistic. For $L<L^{\text {leq }}$, growth is slower and for $L>L^{\text {leq }}$, in general the $\eta$-profiles become unstable (for a small increase, growth may be possible but is then faster than for $L=L^{\text {leq }}$ 
and the molar fractions of Sn at the interface deviate from their equilibrium values). For increasing interface width, growth becomes more sensitive to deviations of $L$ from $L^{\text {leq }}$.

In figure 4, simulation results obtained for the growth of a spherical $\mathrm{Cu}_{6} \mathrm{Sn}_{5}$ grain in a bct matrix supersaturated in $\mathrm{Cu}$ are plotted for different interface widths and for $D_{\mathrm{Cu} 6 \mathrm{Sn} 5}=D_{\mathrm{bct}}$ and $D_{\mathrm{Cu} 6 \mathrm{Sn} 5}=1 \mathrm{e}-5 D_{\mathrm{bct}}$. All curves are for $L=L^{\text {leq }}$. For a given interface width, the kinetic coefficient $L_{1 \text {-sided }}^{\text {leq }}$, calculated for $D_{\mathrm{Cu} 6 \mathrm{Sn} 5}=1 \mathrm{e}-5 D_{\mathrm{bct}}$, is therefore only half of $L_{2 \text {-sided }}^{\text {leq }}$ used for $D_{\mathrm{Cu} 6 \mathrm{Sn} 5}=D_{\mathrm{bct}}$, since $M=0.5 *\left(M_{\mathrm{bct}}+M_{\mathrm{Cu} 6 \mathrm{Sn} 5}\right)$ in equation (25). It was verified that the $\eta$-profiles indeed become unstable for $L=2 L_{1 \text {-sided }}^{\text {leq }}$ when $D_{\mathrm{Cu} 6 \mathrm{Sn} 5} \ll D_{\text {bct }}$. The conditions for the thin interface limit $v_{n} \ell / D \ll 1$ are well obtained within a growth distance $\ell$, since $v_{n} / D=0.5 \cdot 1.6^{2} \cdot\left(x_{\mathrm{bct}, \text { init }}-x_{\mathrm{bct}, \mathrm{eq}}\right)^{2} /\left(x_{\mathrm{Cu} 6 \mathrm{Sn} 5, \mathrm{eq}}-x_{\mathrm{bct}, \mathrm{init}}\right)^{2} \cdot 1 / S \approx 0.009$ for a sphere [49, 53]. For $\ell>R_{\text {init }}$, the shape of the order-parameter profiles deviates from the steady-state profiles, what seriously affects the growth behavior. Also for $\ell<R_{\text {init }}$, there are interface width dependent variations on the velocity and composition at which the $\mathrm{Cu}_{6} \mathrm{Sn}_{5}$ phase forms, during initial growth over a distance of approximately $\ell$ (more than for 1D growth of the intermediate layer). These deviations are related to the fact that the profiles have to become smooth and obtain the speed dictated by bulk diffusion in the bct phase. After this start-up period, however, a parabolic growth regime $R=k \sqrt{t}$ with parabolic growth constant $k=0.04 \mu \mathrm{m} / \mathrm{s}^{1 / 2}$ independent of $\ell$ and $D_{\mathrm{Cu} 6 \mathrm{Sn} 5}$ is reached for $R_{\text {init }}>\ell$ and $\ell / \Delta \mathrm{x}>10$ (using a 5-point stencil for the laplacian), which is close to the theoretically expected growth constant $k \approx 1.6 \sqrt{\frac{x_{\mathrm{bct}, \mathrm{init}}-x_{\mathrm{bct}, \mathrm{eq}}}{x_{\mathrm{Cu} 6 \mathrm{Sn} 5, \mathrm{eq}}-x_{\mathrm{bct}, \mathrm{eq}}}} \sqrt{D_{\mathrm{bct}}}=0.04$ [49, 53]. The composition profiles for 
$D_{\mathrm{Cu} 6 \mathrm{Sn} 5}=1 \mathrm{e}-5 D_{\mathrm{bct}}$ also show that after a growth distance of $\approx 2 \ell$ the $\mathrm{Cu}_{6} \mathrm{Sn}_{5}$ phase forms with a molar fraction of Sn that deviates less than 0.001 from the equilibrium value. For $R_{\text {final }}>4 \ell$, the effect of the initial deviations on the final precipitate size is less than $1 \%$.

Comparison of the composition profiles in figure $4 \mathrm{~b}$ ) reveals that there is a small solute-trapping effect during parabolic growth when $D_{\mathrm{Cu} 6 \mathrm{Sn} 5} \ll D_{\text {bct }}$. The maximum in the composition profiles, reached at a growth distance of approximately $2 \ell$, is namely slightly above the equilibrium composition (the Gibbs-Thomson effect is negligible for the considered particle size and would shift the composition in the other direction). The maximum deviation of the molar fraction field from the equilibrium value is however around 0.001 and diminishes during further growth. The effect on the overall growth behavior and final precipitate size is therefore negligible compared to the deviations introduced during start-up. An antitrapping current [11, 14] would not remove these initialization effects, except that it would allow for the same kinetic coefficient $L^{\text {leq }}$ as for the 2 -sided case. The numerical solution of an antitrapping current however requires a considerable amount of extra calculation time. Nevertheless, to study morphologies with constant growth rate, as for example dendrites and needles, it is still important to extent the antitrapping formalism in a consistent way to multi-component and multi-phase systems with arbitrary thermodynamic and diffusion characteristics. Furthermore, for a general treatment of microstructure evolution with possibly large driving forces and gradients in properties, it remains a main task for the future to verify to which extend thin interface approaches can be relevant to simulate quantitatively local non-equilibrium effects at interfaces or in the 
bulk.

In figure 5, the radius of the shrinking grain during Ostwald ripening of $2 \mathrm{Cu}_{6} \mathrm{Sn}_{5}$ grains in a bct matrix is plotted as a function of time for different simulation conditions. For $\ell / \Delta \mathrm{x}>6.6$, the choice of the interface width has no effect on the evolution of the precipitates as long as the radius of the shrinking grain is larger than $\ell$. When considering Ostwald ripening of a large number of precipitates, the deviations introduced just before disappearance of one of the precipitates, will probably hardly affect the coarsening of the remaining precipitates. The simulations also show that the value of the kinetic coefficient $L$ is far less important for coarsening than for growth.

\section{Conclusions}

A new type of interpolation functions for quantitative phase-field models for multi-phase systems is introduced. Their application within the thin-interface formulation of Tiaden et al. [7] and Kim et al. [9, 14] for multi-component alloys is numerically validated for coarsening and parabolic growth, showing that for the proposed model formulation (1) the bulk energy term and composition fields have no effect on the accuracy with which interfacial energy and triple junction angles are reproduced, (2) the coarsening of precipitates (Ostwald ripening) is not affected by the choice of the diffuse interface width $\ell$ for $R_{\min }>\ell$, with $R_{\min }$ the radius of the smallest precipitate, and (3) for parabolic growth, the effect of interface width becomes negligible and parabolic growth constants are reproduced correctly for $R_{\text {init }}$ or $T_{\text {init }} / 2>\ell$ and $R_{\text {final }}$ or $T_{\text {final }} / 2>4 \ell$, with $R_{\text {init }}$ and $R_{\text {final }}$ the initial and final sphere radius and $T_{\text {init }}$ and $T_{\text {final }}$ the initial and final intermediate layer 
thickness. All conclusions assume that the profiles at the interface are well resolved so that the results are independent of $\Delta \mathrm{x}$.

Besides for chemically driven processes, the interpolation functions will be very valuable in the development of quantitative phase-field approaches coupled with micro-elasticity and plasticity theories [26, 54, 55, 56] for multiphase systems. Interpolation functions with similar properties are required to interpolate in a thermodynamically consistent way the elastic and plastic properties while keeping the minima of the free energy on their intended positions for quantitative accuracy. Furthermore, phenomenological functions that interpolate between the properties of different phases are also used in amplitude equation formulations [19, 57, 58], for which there is currently a renewed interest as a method to coarse-grain phase-field-crystal models or atomic density functional theories.

Acknowledgments: The author is postdoctoral fellow of the Research Foundation - Flanders (FWO-Vlaanderen)

\section{References}

[1] Chen, L.Q.. Annu Rev Mater Res 2002;32:113.

[2] Boettinger, W., Warren, J., Beckermann, C., Karma, A.. Annu Rev Mater Res 2002;23:163.

[3] Singer-Loginova, I., Singer, H.. Rep Prog Phys 2008;71:106501.

[4] Emmerich, H.. Advances in Physics 2008;57:1.

[5] Moelans, N., Blanpain, B., Wollants, P.. Computer Coupling of Phase Diagrams and Thermochemistry (Calphad) 2008;32:268. 
[6] Steinbach, I.. Modelling Simul Mater Sci Eng 2009;17:073001.

[7] Tiaden, J., Nestler, B., Diepers, H., Steinbach, I.. Physica D 1998;115:73.

[8] Eiken, J., Böttger, B., Steinbach, I.. Phys Rev E 2006;73:066122.

[9] Kim, S., Kim, W., Suzuki, T.. Phys Rev E 1999;60:7186.

[10] Karma, A., Rappel, W.J.. Phys Rev E 1996;53:R3017.

[11] Karma, A.. Phys Rev Lett 2001;87:115701.

[12] Echebarria, B., Folch, R., Karma, A., Plapp, M.. Phys Rev E 2004;70:061604.

[13] Ramirez, J., Beckermann, C., Karma, A., Diepers, H.J.. Phys Rev E 2004;69:051607.

[14] Kim, S.. Acta Mater 2007;55:4391.

[15] Steinbach, I., Pezzolla, F., Nestler, B., Seeßelber, M., Prieler, R., Schmitz, G., et al. Physica D 1996;94:135.

[16] Folch, R., Plapp, M.. Phys Rev E 2003;72:011602.

[17] Folch, R., Plapp, M.. Phys Rev E 2005;72:011602.

[18] Chen, L.Q., Yang, W.. Phys Rev B 1994;50:15752.

[19] Khachaturyan, A.G.. Theory of structural transformations in solids. Wiley-interscience publications; Wiley, New York; 1983. ISBN 0-47107873-5. 
[20] Zhu, J., Wang, T., Zhou, S., Liu, Z., Chen, L.. Acta Mater 2004;52:833.

[21] Ofori-Opoku, N., Provatas, N.. Acta Mater 2010;58:2155.

[22] Heulens, J., Moelans, N.. Scripta Mater 2010;62:827.

[23] Moelans, N., Blanpain, B., Wollants, P.. Phys Rev Lett 2008;101:025502.

[24] Moelans, N., Blanpain, B., Wollants, P.. Phys Rev B 2008;78:024113.

[25] Kazaryan, A., Wang, Y., Dregia, S., Patton, B.. Phys Rev B 2000;61:14275.

[26] Steinbach, I., Apel, M.. Physica D 2006;217:153.

[27] Lukas, H., Fries, S., Sundman, B.. Computational thermodynamics: The Calphad Method. Cambridge; 2007. ISBN ISBN 978-0-521-86811-2.

[28] Grafe, U., Böttger, B., Tiaden, J., Fries, S.. Scr Mater 2000;42:1179.

[29] Böttger, B., Eiken, J., Steinbach, I.. Acta Mater 2006;54:2697.

[30] Qin, R., Wallach, E.. Acta Mater 2003;51:6199.

[31] Warnken, N., Ma, D., Drevermann, A., Reed, R., Fries, S., Steinbach, I.. Acta Mater 2009;57:5862.

[32] Zhang, R., Jing, T., Jie, W., Liu, B.. Acta Mater 2006;54:2235.

[33] Wang, G., Xu, D., Ma, N., Zhou, N., Payton, E., Yang, R., et al. Acta Mater 2009;57:316. 
[34] Cha, P.R., Yeon, D.H., Yoon, J.K.. Acta Mater 2001;49:3295.

[35] Wheeler, A., Boettinger, W., McFadden, G.. Phys Rev A 1992;45:7424.

[36] Andersson, J.O., Ȧgren, J.. J Appl Phys 1992;72:1350.

[37] Wu, K., Morral, J., Wang, Y.. Acta Mater 2001;49:3401.

[38] Wu, K., Morral, J., Wang, Y.. Acta Mater 2004;52:1917.

[39] Chen, Q., Ma, N., Wu, K., Wang, Y.. Scr Mater 2004;50:471.

[40] Campbell, C., Boettinger, W., Kattner, U.. Acta Mater 2002;50:775.

[41] Van der Ven, A., Ceder, G.. Phys Rev Lett 2005;94:045901.

[42] Mantina, M., Wang, Y., Chen, L., Liu, Z., Wolverton, C.. Acta Mater 2009;57:4102.

[43] Simonovic, D., Sluiter, M.. Phys Rev B 2009;79:054304.

[44] Van der Ven, A., Thomas, J., Xu, Q., Bhattacharya, J.. Mathematics and computers in simulation 2010;80:1393.

[45] Holm, E., Hassold, G., Miodownik, M.. Acta Mater 2001;49:2981.

[46] Cahn, J., Hilliard, J.. J Chem Phys 1958;28:258.

[47] http://nele.studentenweb.org/docs/parameters.m; http://nele.studentenweb.org/docs/GammaDependence.txt; 2008.

[48] Strandlund, H., Odqvist, J., Ȧgren, J.. Comp Mater Sci 2008;44:265. 
[49] Porter, D., Easterling, K.. Phase Transitions in Metals and Alloys. Chapman \& Hall, New York; 1992.

[50] M, H.. Phase equilibria, phase diagrams and phase transformations : their thermodynamic basis. Cambridge University press; 1998.

[51] Ohno, M., Matsuura, K.. Phys Rev E 2009;79:031603.

[52] Plapp, M.. arXiv:1004.4502v1; 2010.

[53] Zener, C.. J Appl Phys 1949;20:950.

[54] Greenwood, M., Hoyt, J., Provatas, N.. Acta Mater 2009;57:2613.

[55] Vaithyanathan, V., Wolverton, C., Chen, L.. Phys Rev Lett 2002;88:125503.

[56] Gaubert, A., Le Bouar, Y., Finel, A.. Phil Mag 2010;90:375.

[57] Wang, Y., Banerjee, D., Su, C., Khachaturyan, A.. Acta Mater 1998;46:2983.

[58] Spatschek, R., Karma, A.. Phys Rev B 2010;81:214201.

[59] Moelans, N., Wendler, F., Nestler, B.. Comp Mater Sci 2009;46:479. 


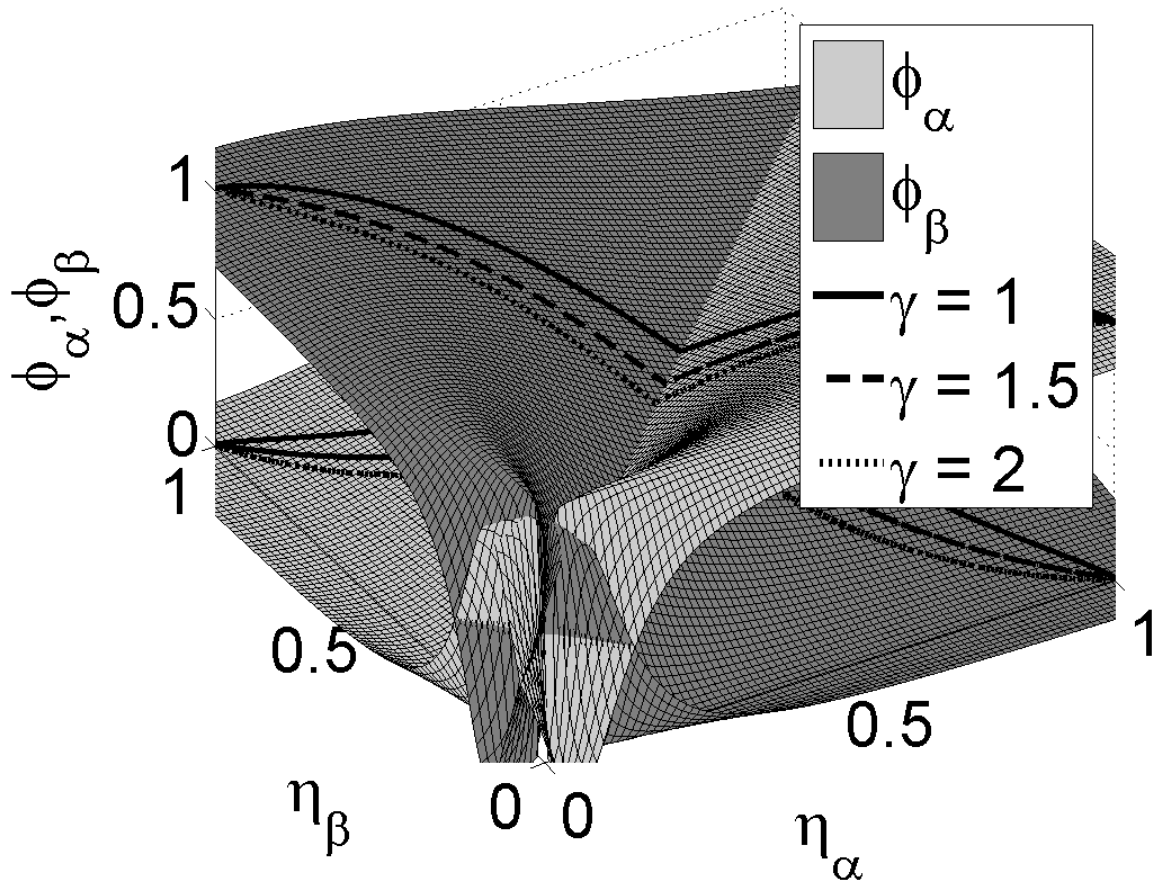

a) 


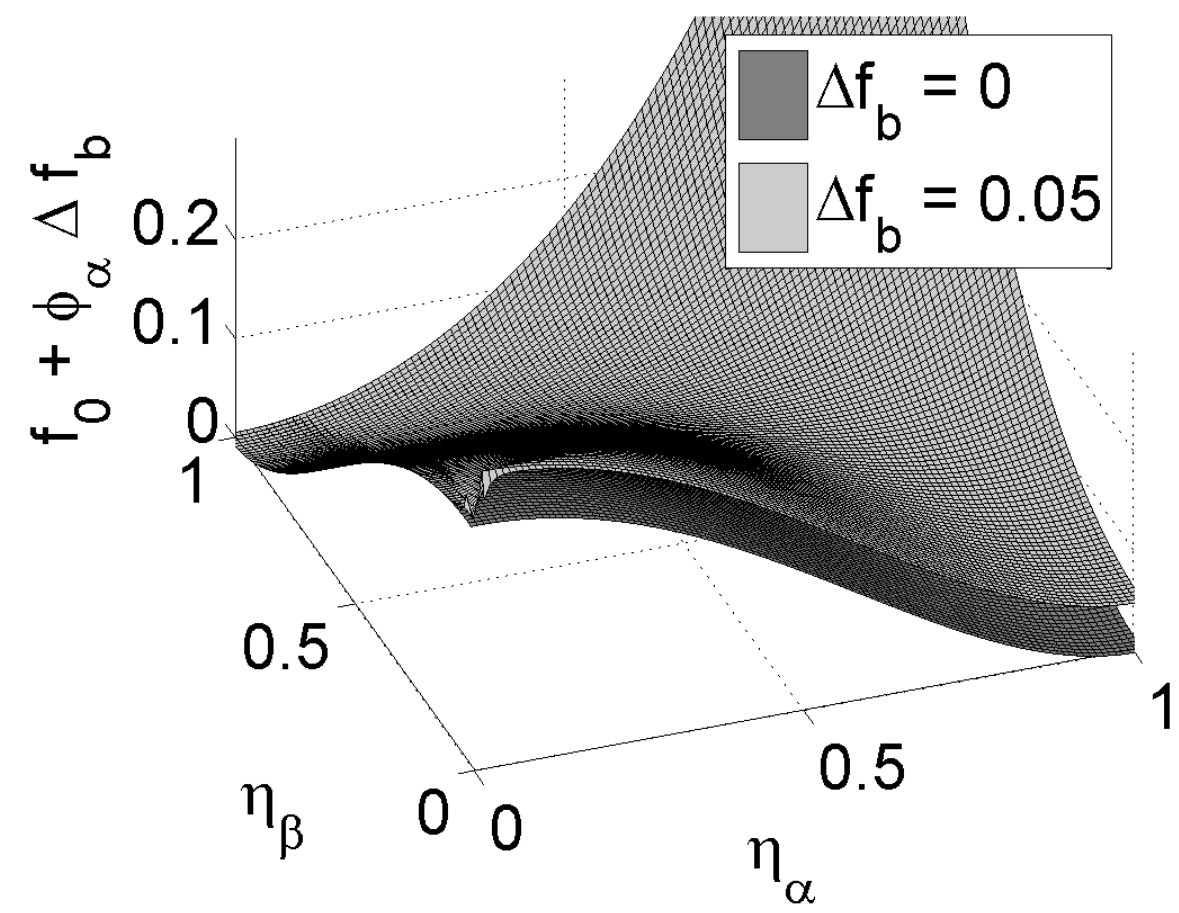

b)

Figure 1: a) Interpolation functions $h_{\alpha}=\phi_{\alpha}=\eta_{\alpha}^{2} /\left(\eta_{\alpha}^{2}+\eta_{\beta}^{2}\right)$ and $h_{\beta}=\phi_{\beta}=\eta_{\beta}^{2} /\left(\eta_{\alpha}^{2}+\eta_{\beta}^{2}\right)$ as a function of the order-parameters for a two-phase system represented by $\eta_{\alpha}$ and $\eta_{\beta}$. The values of $\phi_{\alpha}$ and $\phi_{\beta}$ across an $\alpha / \beta$ interface in local equilibrium is indicated for different values of the model parameter $\gamma$ in the interfacial free energy (4)-(5) (since the $\eta$-profiles depend on $\gamma$, the $\phi$-profiles do as well). The slope of the $\phi$-profiles is always 0 at $\left(\eta_{\alpha}, \eta_{\beta}\right)=(0,1)$ and $(1,0)$. b) Effect of a bulk driving force $\Delta f_{b}\left(f_{\alpha}=\Delta f_{b}\right.$ and $f_{\beta}=0$ ) on the shape of the homogeneous part in the free energy: the local minima remain at $\left(\eta_{\alpha}, \eta_{\beta}\right)=(0,1)$ or $(1,0)$; the bulk free energy of grain $\alpha$ is however shifted with an amount $\Delta f_{b}$ compared to that of grain $\beta$. When growth is controlled by bulk diffusion, $\Delta f_{b} \rightarrow 0$ at the interface and there is thus no effect on the interfacial free energy. 
Table 1: Angles measured from simulations of a stagnated triple junction between 2 $\mathrm{Cu}_{6} \mathrm{Sn}_{5}$ lamella in equilibrium with a bct phase, using the procedure described in 59 . The effect of grain boundary width $\ell$ and grid spacing $\Delta \mathrm{x}$ is studied for different ratios $\sigma_{12}$ to $\sigma_{13}$, resulting in angles $\beta_{c}=103^{\circ}, 120^{\circ}$ and $138^{\circ}$ according to Young's law. The half lamellar spacing is $5 \mu \mathrm{m}$. The parameter relations for the simulations with constant $\ell$ (notfully-variational) are as follows, for $\sigma=0.2: m=1.125 \mathrm{e} 6 / \ell, \kappa=0.200 \mathrm{e}-6 \ell, \gamma=1.1629$; for $\sigma=0.25: m=1.125 \mathrm{e} 6 / \ell, \kappa=0.250 \mathrm{e}-6 \ell, \gamma=1.5$; and $\sigma=0.35: m=1.125 \mathrm{e} 6 / \ell$, $\kappa=0.348 \mathrm{e}-6 \ell, \gamma=2.7149$ (calculated using algorithm 1 from [24] with $\gamma=1.5$ for $\sigma=0.25)$. The parameter relations for the simulations with constant $\kappa$ (fully-variational) are as follows, for $\sigma=0.2: m=2.36 \mathrm{e} 6, \kappa=0.1311 \mathrm{e}-6, \gamma=0.85$; for $\sigma=0.25: m=2.36 \mathrm{e} 6$, $\kappa=0.1311 \mathrm{e}-6, \gamma=1.2$; for $\sigma=0.35: m=2.365 \mathrm{e} 6, \kappa=0.1311 \mathrm{e}-6, \gamma=3.7$ (calculated from (36a)-(36c) of 24]).

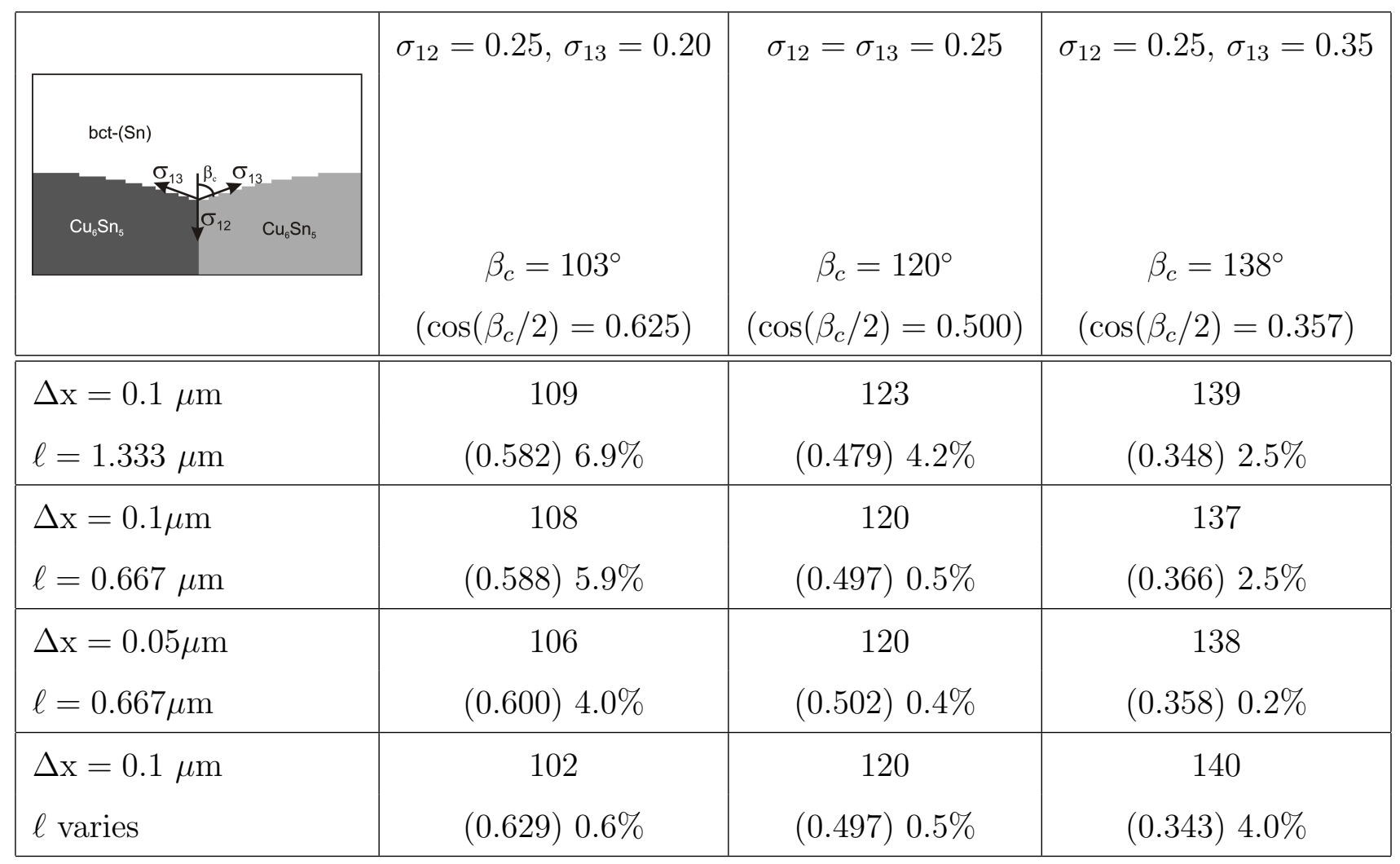




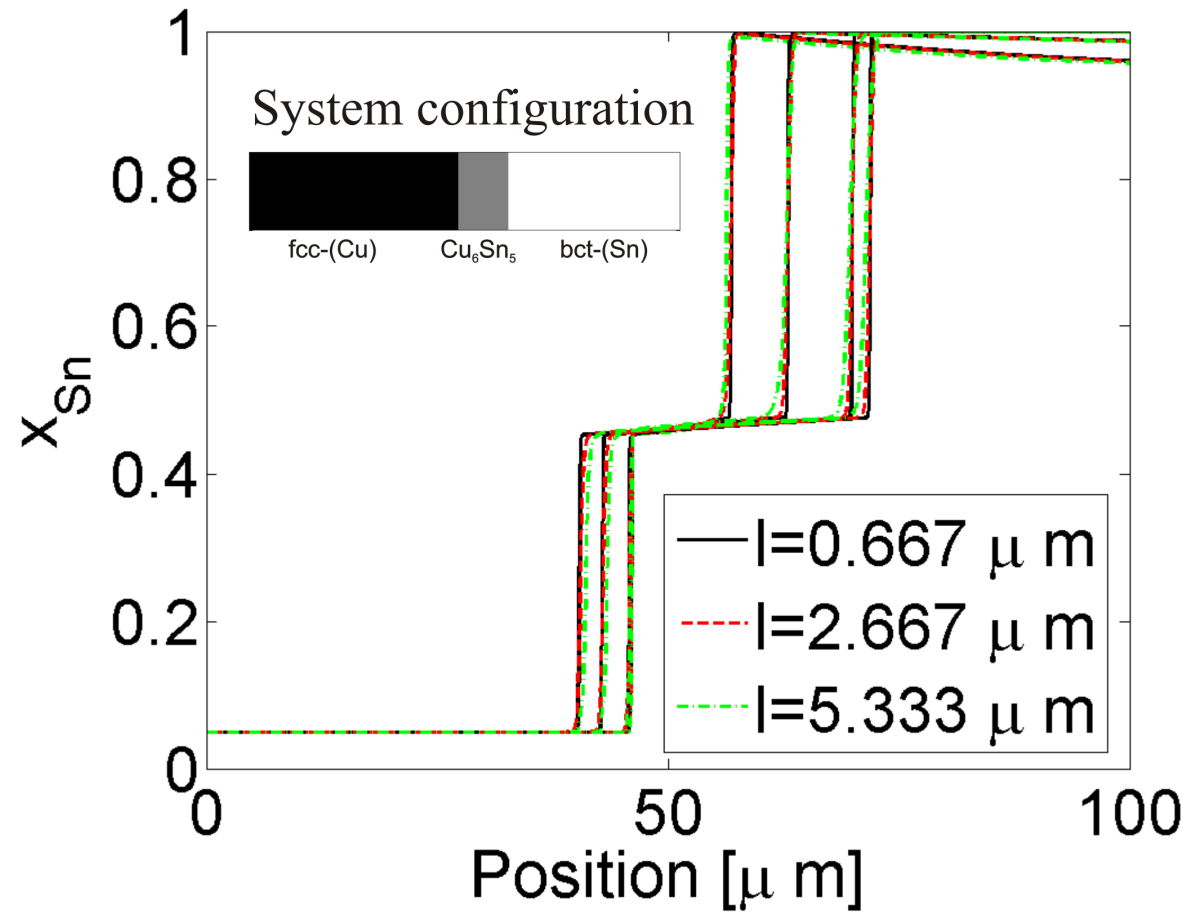

a) 


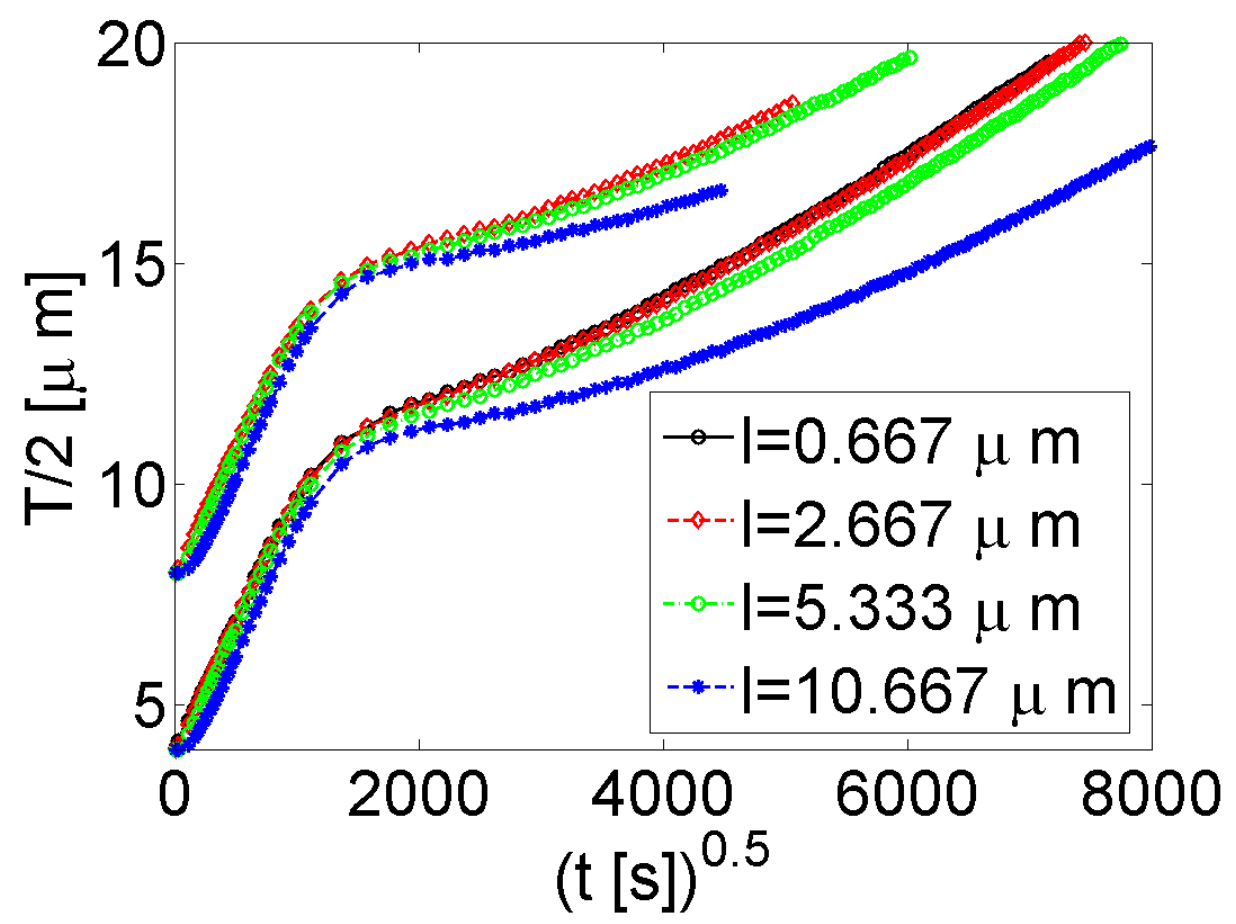

b)

Figure 2: a) Composition profiles at $t=62500,625000,12500000$ and $25000000 \mathrm{~s}$ and b) half intermediate layer thickness $T / 2$ as a function of the square root of time $t$, for different interface widths $\ell$ and $L=L^{\text {leq }}$, obtained from simulations of the growth of an intermediate $\mathrm{Cu}_{6} \mathrm{Sn}_{5}$ phase from fcc $(\mathrm{Cu})$ and bct $(\mathrm{Sn})$ with diffusion coefficients $D_{\mathrm{fcc}}=1 \mathrm{e}-25, D_{\mathrm{Cu} 6 \mathrm{Sn} 5}=1 \mathrm{e}-16$ and $D_{\mathrm{bct}}=1 \mathrm{e}-14\left(\mathrm{~J} / \mathrm{m}^{2}\right)$, initial compositions $x_{\mathrm{fcc}, \text { init }}=0.05$, $x_{\mathrm{Cu6Sn} 5 \text {,init }}=0.455$ and $x_{\mathrm{bct}, \text { init }}=0.95$, grid spacing $\Delta \mathrm{x}=0.1 \mu \mathrm{m}$, system length $200 \mu \mathrm{m}$ (only the left $100 \mu \mathrm{m}$ are plotted) and initial intermediate layer thickness $T_{\text {init }}=8 \mu \mathrm{m}$ or $T_{\text {init }}=16 \mu \mathrm{m}$ initially positioned at $50 \mu \mathrm{m}$. 


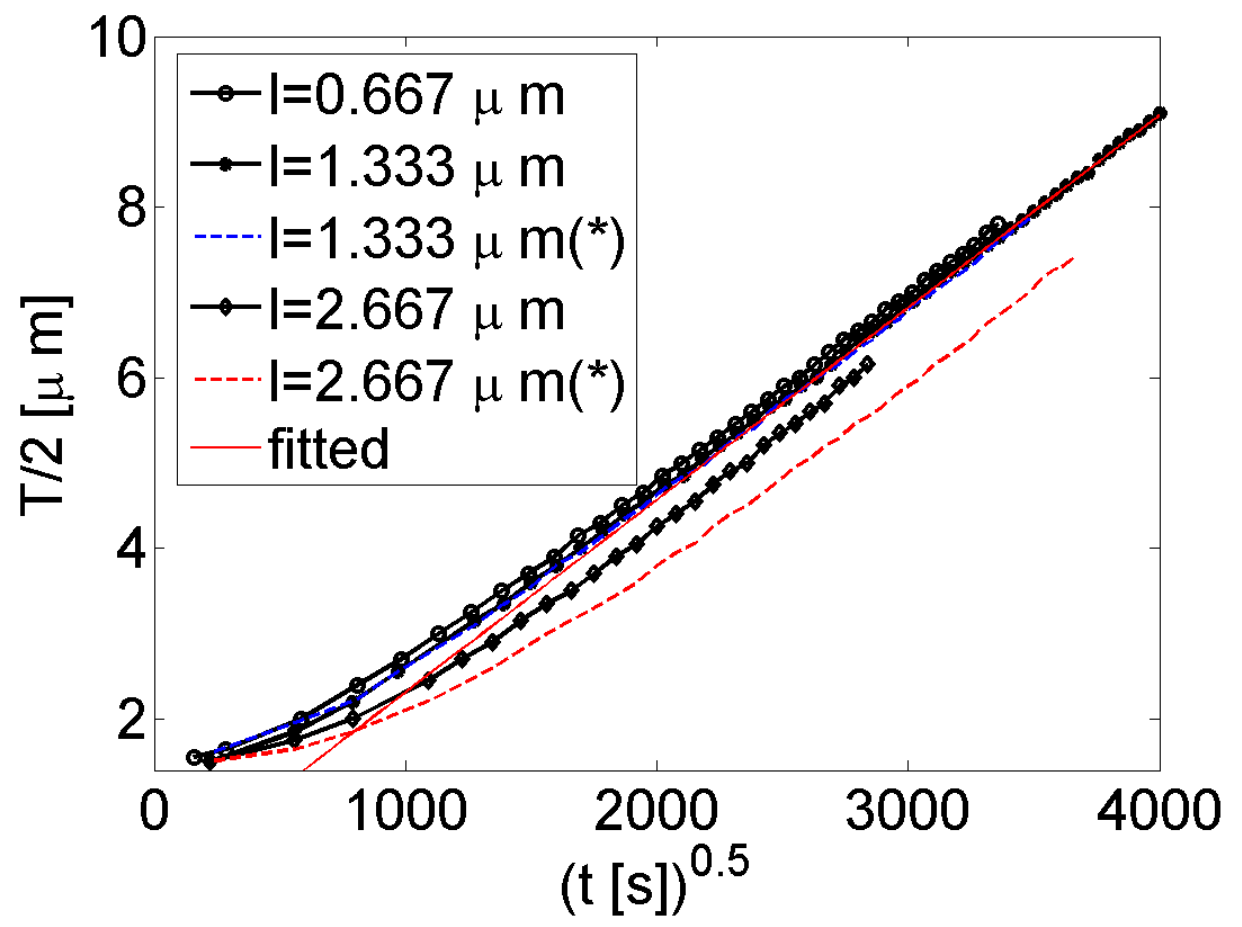

Figure 3: a) Half intermediate layer thickness $T / 2$ as a function of the square root of time $t$ obtained from simulations of the growth of an intermediate $\mathrm{Cu}_{6} \mathrm{Sn}_{5}$ phase from fcc $(\mathrm{Cu})$ and bct $(\mathrm{Sn})$ with diffusion coefficients $D_{\mathrm{fcc}}=1 \mathrm{e}-25, D_{\mathrm{Cu} 6 \mathrm{Sn} 5}=1 \mathrm{e}-16$ and $D_{\mathrm{bct}}=1 \mathrm{e}-13$ $\left(\mathrm{m}^{2} / \mathrm{s}\right)$, initial compositions $x_{\mathrm{fcc}, \text { init }}=0.05, x_{\mathrm{Cu} 6 \mathrm{Sn} 5 \text {,init }}=0.455$ and $x_{\mathrm{bct}, \text { init }}=0.999$, system length $100 \mu \mathrm{m}$, initial intermediate layer thickness $T_{\text {init }}=3 \mu \mathrm{m}$ initially positioned at $50 \mu \mathrm{m}$ and $\Delta \mathrm{x}=0.1 \mu \mathrm{m}$. Results are plotted for different interface widths $\ell$ and $L=L^{\text {leq }}$ and $\left(^{*}\right)$ $L=0.7 L^{\text {leq }}$. For $\ell>3.3 \Delta \mathrm{x}$ and $L=L^{\text {leq }}$, a steady-state regime is reached where $T=k \sqrt{t}$ with $k=0.0045 \mu \mathrm{m} / \mathrm{s}^{1 / 2}$. For $L<L^{\text {leq }}$ initial growth is slower and it takes more time to approach steady-state growth. 


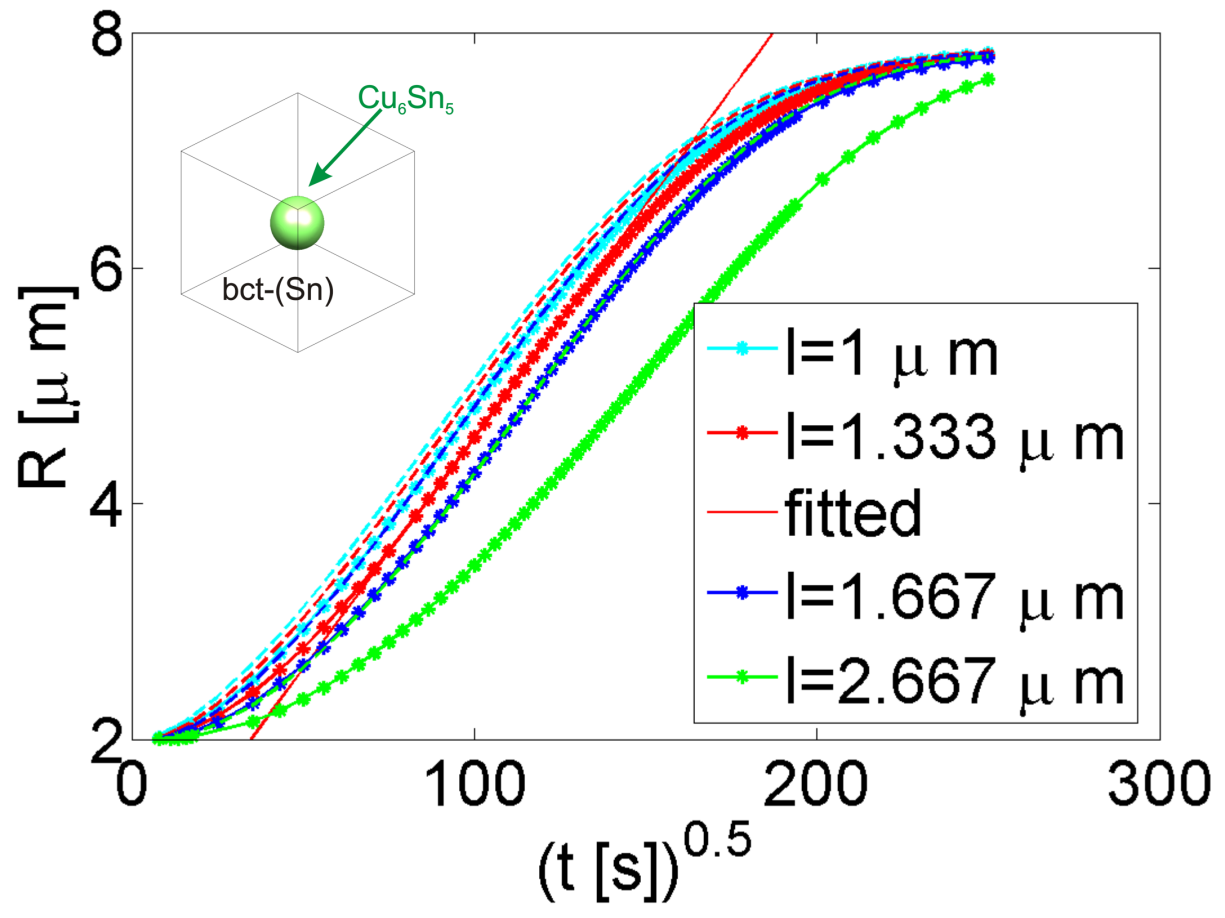

a) 


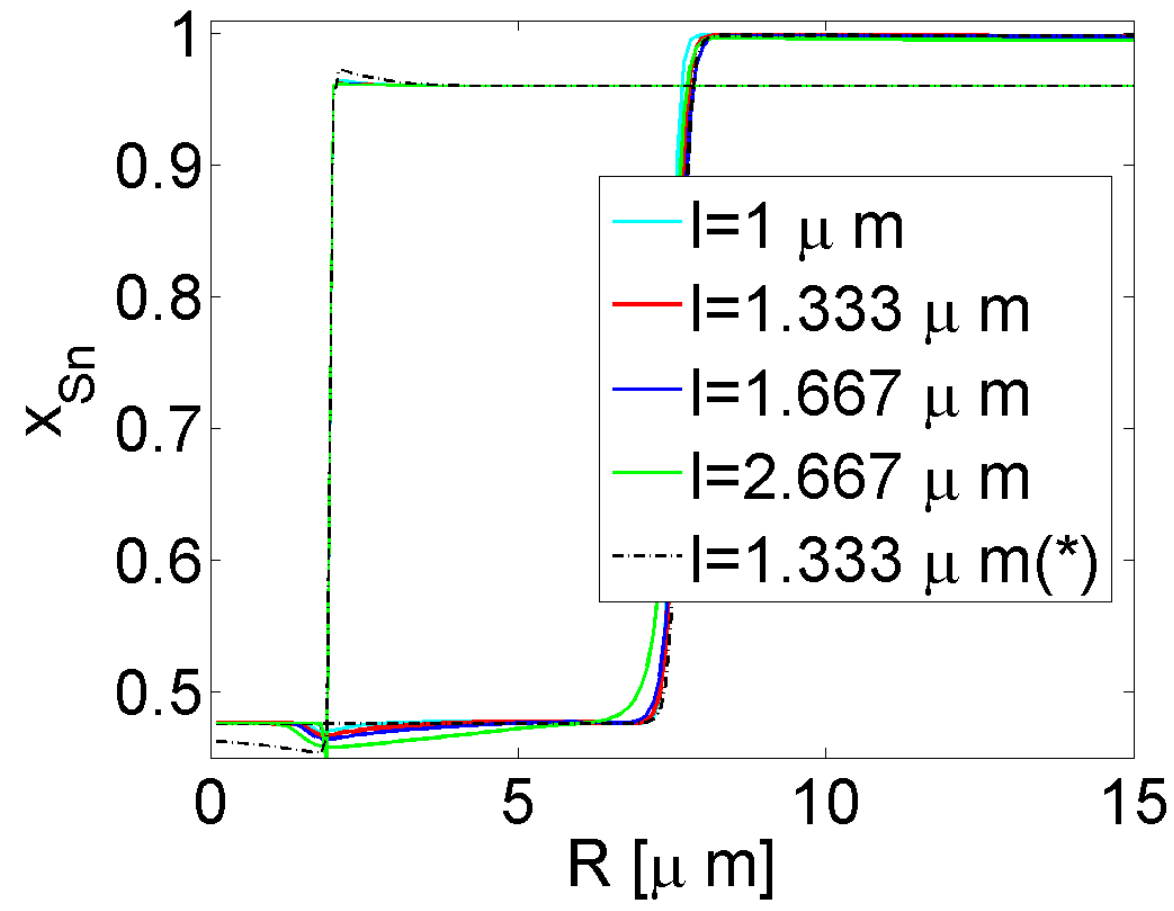

b)

Figure 4: Simulation results obtained for different interface widths $\ell$ from simulations of the growth of a spherical $\mathrm{Cu}_{6} \mathrm{Sn}_{5}$ grain from a supersaturated bct grain with initial compositions $x_{\mathrm{bct}, \text { init }}=0.96$ and $x_{\mathrm{Cu} 6 \mathrm{Sn} 5 \text {,init }}=0.465$, and diffusion coefficients $D_{\mathrm{bct}}=1 \mathrm{e}-14$ and $D_{\mathrm{Cu} 6 \mathrm{Sn} 5}=D_{\mathrm{bct}}$ (broken lines) or $D_{\mathrm{Cu} 6 \mathrm{Sn} 5}=1 \mathrm{e}-5 D_{\mathrm{bct}}$ (full lines), $R_{\text {init }}=2 \mu \mathrm{m}, \Delta \mathrm{x}=0.1$ $\mu \mathrm{m}, L=L^{\text {leq }}$ and system size $30 \times 30 \times 30 \mu \mathrm{m}^{3}$. a) Radius $R$ as a function of the square root of time $t$. For $\ell / \Delta \mathrm{x}>10$ and $R_{\text {final }}>4 \ell$ a parabolic growth regime is reached where $R=k \sqrt{t}$ with $k=0.04 \mu \mathrm{m} / \mathrm{s}^{1 / 2}$. b) Composition profiles at $\mathrm{t}=62.5$ and $62500 \mathrm{~s}$ for different interface widths and $D_{\mathrm{Cu} 6 \mathrm{Sn} 5}=1 \mathrm{e}-5 D_{\mathrm{bct}}$ and for $\ell=1.33 \mu \mathrm{m}$ and $D_{\mathrm{Cu} 6 \mathrm{Sn} 5}=D_{\mathrm{bct}}$. Because of the large difference in diffusivities for $D_{\mathrm{Cu} 6 \mathrm{Sn} 5}=1 \mathrm{e}-5 D_{\mathrm{bct}}$, the composition at which the $\mathrm{Cu}_{6} \mathrm{Sn}_{5}$ phase forms is maintained during growth, whereas for $D_{\mathrm{Cu} 6 \mathrm{Sn} 5}=D_{\text {bct }}$ the composition of the precipitate homogenizes continuously during growth. 


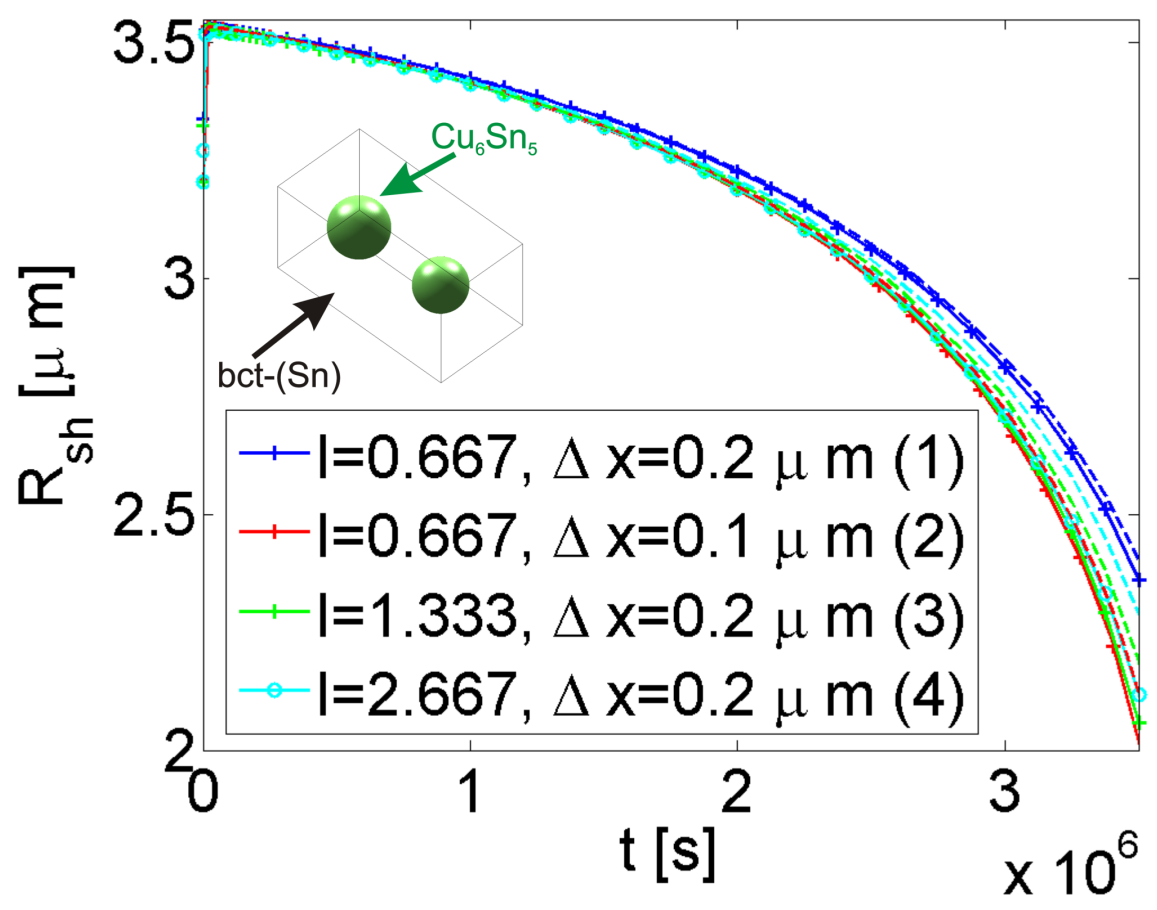

Figure 5: Radius as a function of time of the shrinking grain obtained from a simulation of Ostwald ripening of $2 \mathrm{Cu}_{6} \mathrm{Sn}_{5}$ grains in a bct matrix with diffusion coefficients $D_{\text {bct }}=1 \mathrm{e}-$ $14 \mathrm{~m}^{2} / \mathrm{s}$ and $D_{\mathrm{Cu} 6 \mathrm{Sn} 5}=D_{\mathrm{bct}}$ (full lines) or $D_{\mathrm{Cu} 6 \mathrm{Sn} 5}=1 \mathrm{e}-19 \mathrm{~m}^{2} / \mathrm{s}$ (broken lines), initial compositions are $x_{\mathrm{Cu} 6 \mathrm{Sn} 5 \text {,init }}=0.455$ and $x_{\mathrm{bct}, \text { init }}=0.99$, initial radii $R_{\mathrm{sh}, \text { init }}=3.2 \mu \mathrm{m}$ and $R_{\mathrm{gr}, \text { init }}=3.6 \mu \mathrm{m}$ and system size $28 \times 14 \times 14 \mu \mathrm{m}^{3}$. Curves are plotted for different interface widths $\ell$ and grid spacings $\Delta \mathrm{x}$ and for $L \approx L^{\text {leq }}$, namely $L=0.34 L^{\text {leq }}(1-2), L=0.68 L^{\text {leq }}$ (3), $L=L^{\text {leq }}(4)$. For curve (1), the numerical resolution $\ell / \Delta \mathrm{x}$ is too low. 